\title{
Weekly Cisplatin-Based Concurrent Chemoradiotherapy for Treatment of Locally Advanced Head and Neck Cancer: a Single Institution Study
}

\author{
Saptarshi Ghosh*, Pamidimukkala Brahmananda Rao, P Ravindra Kumar, \\ Surendra Manam
}

\begin{abstract}
Background: The organ preservation approach of choice for the treatment of locally advanced head and neck cancers is concurrent chemoradiation with three weekly high doses of cisplatin. Although this is an efficacious treatment policy, it has high acute systemic and mucosal toxicities, which lead to frequent treatment breaks and increased overall treatment time. Hence, the current study was undertaken to evaluate the efficacy of concurrent chemoradiation using $40 \mathrm{mg} / \mathrm{m} 2$ weekly cisplatin. Materials and Methods: This is a single institutional retrospective study including the data of 266 locally advanced head and neck cancer patients who were treated with concurrent chemoradiation using $40 \mathrm{mg} / \mathrm{m} 2$ weekly cisplatin from January 2012 to January 2014. A p-value of $<\mathbf{0 . 0 5}$ was taken to be significant statistically for all purposes in the study. Results: The mean age of the study patients was 48.8 years. Some $36.1 \%$ of the patients had oral cavity primary tumors. The mean overall treatment time was $\mathbf{5 7 . 2}$ days. With a mean follow up of $\mathbf{1 5 . 2}$ months for all study patients and $\mathbf{1 7 . 5}$ months for survivors, 3 year local control, locoregional control and disease free survival were seen in $62.8 \%, 42.8 \%$ and $42.1 \%$ of the study patients. Primary tumor site, nodal stage of disease, AJCC stage of the disease and number of cycles of weekly cisplatin demonstrated statistically significant correlations with 3 year local control, locoregional control and disease free survival. Conclusions: Concurrent chemoradiotherapy with moderate dose weekly cisplatin is an efficacious treatment regime for locally advanced head and neck cancers with tolerable toxicity which can be used in developing countries with limited resources.
\end{abstract}

Keywords: Head and neck cancer - organ preservation treatment - chemoradiation - cisplatin dosage

Asian Pac J Cancer Prev, 16 (16), 7309-7313

\section{Introduction}

Some $57.5 \%$ of the worldwide head and neck cancers (excluding oesophageal cancers) occur in Asia, especially in India (Chaturvedi, 2009). The reported burden of head and neck cancers (HNCA) in India is actually much less and is analogous to the tip of the iceberg (Mishra et al., 2014). Most of the patients with HNCA present in Stage IV disease in India (Pandey et al., 2014).

Chemoradiotherapy based organ preservation approach is currently the standard of care for the management of HNCA (Lasrado et al., 2014). According to the meta-analysis of the MACH-NC collaborative group (Pignon et al., 2007), which demonstrated a 6.5\% absolute survival advantage at 5 years in HNCA with concurrent chemoradiation, platinum based concurrent chemoradiation has become the non-surgical standard of care in the treatment of locally advanced head and neck cancers (LAHNC). Although 3 weekly high dose cisplatin is the current chemotherapy of choice while given concurrently with radiation (Pignon et al., 2000; Pignon et al., 2005; Pignon et al., 2007), there is still no uniform consensus due to the wide variation in various study designs and due to the different ways of combining chemotherapy with radiation (Browman et al., 2001; Baykara et al., 2013). Also the 3 weekly high dose cisplatin based chemoradiation regime has been shown to have considerable mucosal and haematological toxicity and is non-compliant in one-third of the patients (Brizel et al., 2006). The situation is further complex in developing countries like India, where there are limited resources and the patients need intensive inpatient care for the acute toxicities. Hence, moderate doses of weekly cisplatin has been advocated as concurrent chemotherapy (Dimri et al., 2013) while the patient is on radiation.

The current study aims at finding out the efficacy of moderate dose weekly cisplatin based concurrent chemoradiation in Indian setting. 


\section{Materials and Methods}

\section{Study design}

This is a single institutional retrospective study done with the data available from the computer and clinical case sheets of the patients with locally advanced squamous cell carcinoma of the head and neck who were treated in the Department of Radiotherapy in a South Indian institute from January 2012 to January 2014. Previously untreated patients with non-metastatic LAHNC (excluding cancers of the esophagus, nasopharynx and paranasal sinuses) who were treated with $40 \mathrm{mg} / \mathrm{m}^{2}$ cisplatin based concurrent chemoradiation were only included in the study. Patients treated with neoadjuvant or adjuvant chemotherapy were excluded from the study. Patients treated with any other chemotherapeutic agents or with any other dosage regime of cisplatin were also excluded from the study. All patients who completed their treatment were only included in the present study. A total of 266 patients were found to be satisfying the inclusion criteria of the study.

\section{Radiotherapy}

All patients were treated with conventional radiotherapy with $6 \mathrm{MV}$ photons with a linear accelerator after immobilization with thermoplastic mask. All patients were treated with conventional fractionation. The gross tumor volume was treated to a dose of 66 or $70 \mathrm{~Gy}$ in 33 - 35 fractions. The first phase $46 \mathrm{~Gy}$ was delivered in 23 fractions to the mid-plane with pair opposing lateral fields. The lower neck was treated with a matched low anterior neck field upto a dose of 50 Gy in 25 fractions using a half beam block, normalized at $2-3 \mathrm{cms}$ of depth. In the second phase, shrinking field off-cord technique was used to deliver the radiation dose to the primary tumor site along with the nodal sites with a 2-3 cms margin upto a dose of 66 or $70 \mathrm{~Gy}$.

\section{Chemotherapy}

$40 \mathrm{mg} / \mathrm{m}^{2}$ weekly cisplatin was administered intravenously during the course of radiation. Prechemotherapy hydration was given along with standard antiemetic premedication. Cisplatin was delivered along with $500 \mathrm{ml}$ of normal saline over 3 hours. Postchemotherapy hydration was given along with $10 \mathrm{ml}$ of magnesium sulphate. $100 \mathrm{ml}$ of $20 \%$ mannitol was given post-chemotherapy to ensure forced diuresis. On the day of the chemotherapy, radiation was delivered within an hour of cisplatin administration. Post-chemotherapy antiemetic prophylaxis was given. Chemotherapy administration was postponed if the haemoglobin level was less than 9 $\mathrm{gm} / \mathrm{dl}$ or total leukocyte count was less than $2500 \mathrm{~mm}-3$ or platelet count was less than $75,000 \mathrm{~mm}-3$ or serum creatinine was more than $1.5 \mathrm{mg} \%$, till recovery. Dose modifications were not done in any patient.

\section{Patient evaluation}

All the study patients were evaluated weekly during the course of chemoradiotherapy to assess the toxicity of the treatment. After completion of chemoradiation, all patients were followed up monthly for the next two months, then every 3-6 monthly for the next 2 years and annually thereafter. All acute toxicities were recorded according to the Radiation Therapy Oncology Group (RTOG) guidelines (Cox et al., 1995). Disease control was assessed clinically and with direct laryngoscopy whenever indicated. Patients who did not turn for follow up were considered lost to follow up and were censored for statistical analysis.

\section{Statistical analysis}

Local failure was defined as persistence or reappearance of disease at or in close proximity to the primary tumor site. Locoregional failure was defined as persistence or reappearance of the disease either at the primary site or in draining regional lymph nodes. Relapse was defined as recurrence of disease at either the primary, regional or distant site or the appearance of a second primary in the upper aero-digestive tract. All data were tabulated in Microsoft Excel and analysed with SPSS (Statistical Package for Social Sciences) software version 20. The local control, loco-regional control and disease free survival were calculated using the Kaplan-Meier method. All the estimates were calculated from the date of initiation of treatment till the defined event if any or until last contact or death. The analysis was limited only to first failures as a measure of efficacy of concurrent chemoradiotherapy and does not include the outcome after attempted salvage treatments. The data was compared using the Log-rank test for univariate analysis. $\mathrm{p}$-value $<0.05$ was taken to be statistically significant.

\section{Results}

The mean age of the study patients was 48.8 years. $59 \%$ of the study patients were of age less than equal to 50 years. $36.1 \%$ of the study patients had primary oral cavity tumors, mostly of the hard palate and buccal mucosa. $31.2 \%, 17.3 \%$ and $15.4 \%$ patients had primary tumors in the oropharynx, hypopharynx and larynx respectively. $53.8 \%$ patients had T3 tumors. Majority of the patients had $\mathrm{N} 2$ disease. $54.5 \%$ of the study patients had AJCC Stage IV tumors at diagnosis. $65.4 \%$ of the patients received $66 \mathrm{~Gy}$ dose of radiation, while rest of the study patients received $70 \mathrm{~Gy}$. Majority of the study patients (78.2\%) received more than 4 cycles of weekly cisplatin (Table 1). The mean overall treatment time of the study patients was 57.2 days. $54.5 \%$ patients completed their treatment within 57 days (Table 1).

\section{Local control}

With a mean follow up of 15.2 months for all study patients and 17.5 months for survivors, the 3 year local control was $62.8 \%$. Primary tumor site $(<0.001)$ (Figure $1 \mathrm{a})$, T stage $(<0.001)$, nodal stage $(<0.001)$, AJCC tumor stage (0.014) (Figure 2a), cycles of concurrent weekly cisplatin $(<0.001)$ and overall treatment time $(0.009)$ showed statistically significant association with local tumor control in the study patients (Table 1).

\section{Locoregional control}

The 3 year locoregional control of the study patients was $42.8 \%$. Primary tumor site $(<0.001)$ (Figure $1 b), \mathrm{N}$ 
Weekly Cisplatin-Based Concurrent Chemoradiotherapy for the Treatment of Locally Advanced Head and Neck Cancer Table 1. Univariate Analysis of the Prognostic Factors for 3 Year Local Control, Loco-Regional Control and Overall Control in the Study Patients

\begin{tabular}{|c|c|c|c|c|c|c|c|}
\hline Prognostic Factor & $\begin{array}{c}\text { Number of } \\
\text { Patients }(\%)\end{array}$ & $\begin{array}{c}3 \text { year Local } \\
\text { Control \% }\end{array}$ & $\mathrm{p}$ - value & $\begin{array}{c}3 \text { year Locoregional } \\
\text { Control \% }\end{array}$ & p- value & $\begin{array}{c}3 \text { year Overall } \\
\text { Control \% }\end{array}$ & p-value \\
\hline \multicolumn{8}{|l|}{ Age } \\
\hline$\leq 50$ years & $157(59 \%)$ & $63.60 \%$ & \multirow[t]{2}{*}{0.677} & $41.00 \%$ & \multirow[t]{2}{*}{0.246} & $40.50 \%$ & \multirow[t]{2}{*}{0.342} \\
\hline$>50$ years & $109(41 \%)$ & $62.90 \%$ & & $46.90 \%$ & & $46.00 \%$ & \\
\hline \multicolumn{8}{|l|}{ Primary tumor site } \\
\hline Oral cavity & $96(36.1 \%)$ & $52.50 \%$ & \multirow[t]{4}{*}{$<0.001$} & $34.60 \%$ & \multirow[t]{4}{*}{$<0.001$} & $34.80 \%$ & \multirow[t]{4}{*}{$<0.001$} \\
\hline Oropharynx & $83(31.2 \%)$ & $61.40 \%$ & & $41.10 \%$ & & $40.10 \%$ & \\
\hline Hypopharynx & $46(17.3 \%)$ & $59.70 \%$ & & $37.90 \%$ & & $38.20 \%$ & \\
\hline Larynx & $41(15.4 \%)$ & $93.50 \%$ & & $73.20 \%$ & & $70.40 \%$ & \\
\hline \multicolumn{8}{|l|}{ T Stage } \\
\hline $\mathrm{T} 1$ & $20(7.5 \%)$ & $100 \%$ & \multirow[t]{4}{*}{$<0.001$} & $27.70 \%$ & \multirow[t]{4}{*}{0.052} & $25.70 \%$ & \multirow[t]{4}{*}{0.088} \\
\hline $\mathrm{T} 2$ & $60(22.6 \%)$ & $69 \%$ & & $38.00 \%$ & & $39.20 \%$ & \\
\hline T 3 & $143(53.8 \%)$ & $63.30 \%$ & & $47.90 \%$ & & $46.90 \%$ & \\
\hline $\mathrm{T} 4$ & $43(16.2 \%)$ & $41.60 \%$ & & $39.40 \%$ & & $39.60 \%$ & \\
\hline \multicolumn{8}{|l|}{ N Stage } \\
\hline $\mathrm{No}$ & $51(19.2 \%)$ & $67.20 \%$ & \multirow[t]{4}{*}{$<0.001$} & $57.30 \%$ & \multirow[t]{4}{*}{$<0.001$} & $57.30 \%$ & \multirow[t]{4}{*}{$<0.001$} \\
\hline N 1 & $86(32.3 \%)$ & $65.70 \%$ & & $47.40 \%$ & & $46.90 \%$ & \\
\hline $\mathrm{N} 2$ & $103(38.7 \%)$ & $67.60 \%$ & & $38.20 \%$ & & $36.80 \%$ & \\
\hline N 3 & $26(9.8 \%)$ & $33.90 \%$ & & $11.50 \%$ & & $11.50 \%$ & \\
\hline \multicolumn{8}{|l|}{ AJCC Stage } \\
\hline Stage III & $121(45.5 \%)$ & $66.70 \%$ & \multirow[t]{2}{*}{0.014} & $49.80 \%$ & \multirow[t]{2}{*}{0.018} & $49.50 \%$ & \multirow[t]{2}{*}{0.016} \\
\hline Stage IV & $145(54.5 \%)$ & $61.90 \%$ & & $36.80 \%$ & & $35.80 \%$ & \\
\hline \multicolumn{8}{|l|}{ RT Dose } \\
\hline $66 \mathrm{~Gy}$ & $174(65.4 \%)$ & $56.90 \%$ & \multirow[t]{2}{*}{0.705} & $35.20 \%$ & \multirow[t]{2}{*}{0.085} & $34.30 \%$ & \multirow[t]{2}{*}{0.054} \\
\hline $70 \mathrm{~Gy}$ & $92(34.6 \%)$ & $70.50 \%$ & & $56.70 \%$ & & $56.80 \%$ & \\
\hline \multicolumn{8}{|c|}{ Cycles of Chemotherapy } \\
\hline$\leq 4$ & $58(21.8 \%)$ & $42.70 \%$ & \multirow[t]{2}{*}{$<0.001$} & $21.10 \%$ & \multirow[t]{2}{*}{$<0.001$} & $18.50 \%$ & \multirow[t]{2}{*}{$<0.001$} \\
\hline$>4$ & $208(78.2 \%)$ & $68.40 \%$ & & $49.60 \%$ & & $49.10 \%$ & \\
\hline \multicolumn{8}{|c|}{ Overall treatment time } \\
\hline$\leq 57$ days & $145(54.5 \%)$ & $72.70 \%$ & \multirow[t]{2}{*}{0.009} & $50.00 \%$ & 0.05 & $48.40 \%$ & 0.089 \\
\hline$>57$ days & $121(45.5 \%)$ & $45.30 \%$ & & $29.90 \%$ & & $30.10 \%$ & \\
\hline
\end{tabular}
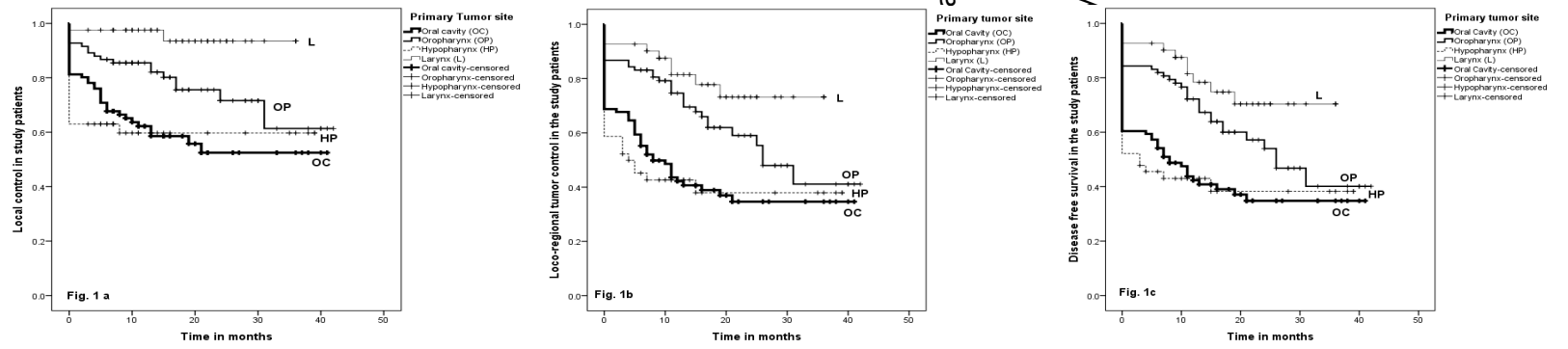

Figure 1. Primary Tumor Site as a Prognostic Factor for Local Control (1a), Loco-regional Control (1b) and Disease Free Survival (1c) in LAHNC Treated with Moderate dose weekly Cisplatin Based Concurrent Chemoradiotherapy

stage $(<0.001)$, AJCC tumor stage $(0.018)$ (Figure $2 \mathrm{~b})$ and number of cycles of chemotherapy $(<0.001)$ demonstrated significant statistical correlation with the locoregional tumor control (Table 1). Overall treatment time showed an almost significant statistical association ( $\mathrm{p}$-value 0.05 ) with locoregional control of tumor (Table 1).

\section{Disease free survival}

The 3 year disease free survival of the study patients was $42.1 \%$. Primary tumor site $(<0.001)$ (Figure $1 \mathrm{c}), \mathrm{N}$ stage $(<0.001)$, AJCC tumor stage $(0.016)$ (Figure $2 \mathrm{c})$ and number of cycles of chemotherapy $(<0.001)$ demonstrated significant statistical correlation with the disease free survival (Table 1).
Acute toxicity

Radiation Therapy Oncology Group (RTOG) Grade III or IV acute toxicities like mucositis, dermatitis, emesis, and haematological toxicity were seen in $48.1 \%, 36.8 \%$, $7.1 \%$, and $7.9 \%$ of the patients respectively.

\section{Treatment breaks}

This study gives a true picture of rural India, where inadvertent treatment breaks are created in 34.2\% (91) of the study patients due to the lack of knowledge and reluctance of the patients and their attenders, thereby prolonging the overall treatment time. $27.4 \%$ of the study patients experienced treatment breaks due to acute toxicities. 

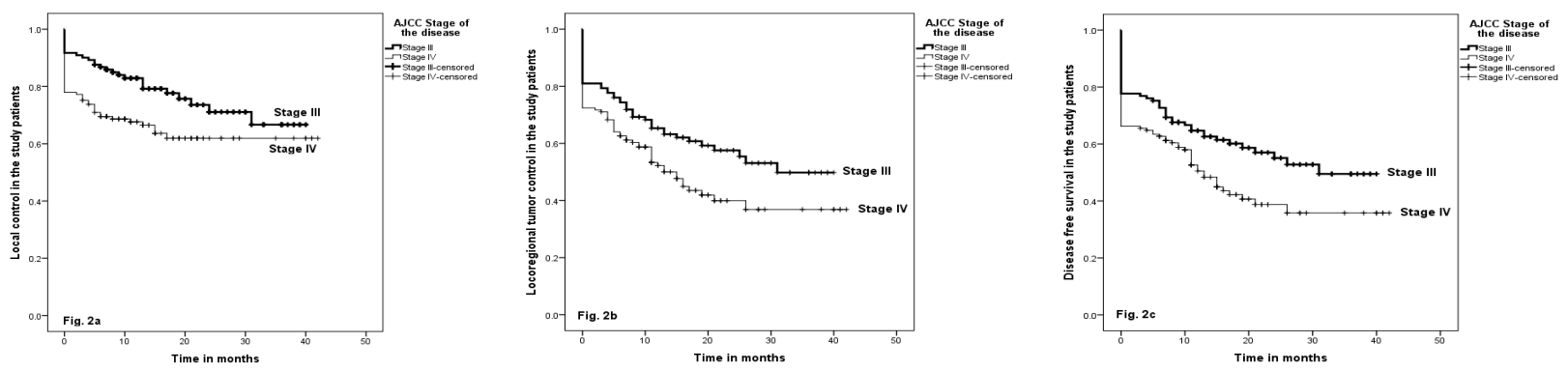

Figure 2. AJCC Tumor Stage as a Prognostic Factor for Local Control (2a), Loco-regional Control (2b) and Disease Free Survival (2c) in LAHNC Treated with Moderate Dose Weekly Cisplatin Based Concurrent Chemoradiotherapy

\section{Discussion}

The present study reports one of the largest singleinstitutional experience of concurrent weekly cisplatin based chemoradiation in the non-surgical management of LAHNC in South India. The mean age of presentation with LAHNC in our study was 48.8 years. This is quite low when compared to western literature (Fan et al., 2012). But similar mean age of presentation has been reported in Indian studies (Gupta et al., 2009; Dimri et al., 2013). The earlier age of presentation in the current study may be attributed to the habit of tobacco chewing, smoking and also reverse smoking. The most commonly encountered primary tumors were that of oral cavity (hard palate mostly), which can be again attributed to the practice of reverse smoking in this part of the country and also the habit of chewing tobacco and betelnut. The mean overall treatment time was higher in the current study when compared to other literature, because most of the present study patients were illiterate and came from rural background, hence were at times reluctant to come for treatment even after repeated counselling.

A meta-analysis (Pignon et al., 2009) of 87 trials demonstrated a $4.5 \%$ absolute benefit of chemotherapy at 5 years. Also, both direct and indirect comparisons demonstrated a more pronounced benefit with concurrent chemotherapy when compared to induction chemotherapy. The absolute benefit was $6.5 \%$ at 5 years. Cisplatin acts synergistically with radiation in the treatment of LAHNC in the following ways - as a radiosensitizer by inhibiting potentially lethal and sublethal damage repair, as a hypoxic cell sensitizer, as a cell cycle inhibitor, by forming deoxyribonucleic acid adducts and as an inhibitor of angiogenesis (Marcu et al., 2003). The use of 3 weekly cisplatin at $100 \mathrm{mg} / \mathrm{m}^{2}$ concurrently with radiation is now recommended in the non-surgical management of LAHNC for organ preservation (Adelstein et al., 2003; Forastiere et al., 2003; Pignon et al., 2007). But only 60\% of the patients actually remain fit enough to receive the desired 3 cycles of chemotherapy due to higher systemic and mucosal toxicities (Brizel et al., 2006). The noncompliance is even more in developing countries due to the limited resources (Dimri et al., 2013). Few studies have demonstrated moderate efficacy of weekly $40 \mathrm{mg} /$ $\mathrm{m}^{2}$ cisplatin based chemoradiotherapy with less toxicity and better patient compliance, especially in developing countries with limited resources (Gupta et al., 2009; Dimri et al., 2013).
In the present study, the 3 year local control, locoregional control and disease free survival was seen in $62.8 \%, 42.8 \%$ and $42.1 \%$ of the study patients. Gupta et al. (2009) found a 5 year local control, locoregional control, and disease free survival of $57 \%, 46 \%$, and $43 \%$ respectively. Another study (Dimri et al., 2013), found a 5 year disease free survival of $39.4 \%$. As most of the disease recurrences and relapses in LAHNC occur within the first three years of treatment, the result of the current study is quite in concordance with the other studies from India.

Alike an Indian institutional experience (Gupta et al., 2009), the present study also demonstrated a significant association of local tumor control with primary tumor site, T stage, AJCC stage of the disease, number of cycles of chemotherapy and overall treatment time. Also in the present study, the nodal stage of the disease, AJCC Stage of the tumor, primary tumor site and number of cycles of chemotherapy administered concurrently with radiation demonstrated significant correlation with locoregional tumor control and disease free survival, similar to a study from Mumbai (Gupta et al., 2009).

In the laryngeal preservation trial by Forastiere et al. (Forastiere et al., 2003), only 70\% patients received the desired 3 cycles of high dose cisplatin during radiation. Also Grade III or IV pharyngitis, haematological toxicity, emesis and nephrotoxicity were seen in $78 \%, 47 \%, 20 \%$ and $4 \%$ of the study patients respectively. Whereas, a study (Dimri et al., 2013) from India with weekly $35 \mathrm{mg} /$ $\mathrm{m}^{2}$ cisplatin based chemoradiation demonstrated acute Grade III or IV mucositis, emesis, anemia and leukopenia in $67 \%, 3 \%, 0.5 \%$ and $0.5 \%$ patients respectively. Another study (Gupta et al., 2009) demonstrated acute Grade III or IV mucositis and dermatitis in only $29 \%$ and $35 \%$ of the study patients respectively. Similarly, in the present study acute Grade III or IV mucositis, dermatitis, emesis and haematological toxicities were seen in $48.1 \%, 36.8 \%$, $7.1 \%$, and $7.9 \%$ of the study patients respectively. Even though most of the patients were from rural background and lacked proper nutrition, the high grade toxicities were quite comparable to other regional studies. Another study (Geeta et al., 2006) with $40 \mathrm{mg} / \mathrm{m}^{2}$ cisplatin based chemoradiotherapy demonstrated an overall treatment compliance of $65 \%$, significant hematologic toxicity of around $20 \%$ and treatment related hospital admissions of approximately $30 \%$.

In conclusion, the treatment of LAHNC with $40 \mathrm{mg} / \mathrm{m}^{2}$ weekly cisplatin based chemoradiation is an effective and 
Weekly Cisplatin-Based Concurrent Chemoradiotherapy for the Treatment of Locally Advanced Head and Neck Cancer less toxic regime. It can be especially used in developing nations with limited resources, where intensive in-patient care throughout the period of chemoradiation is hardly possible. The patient compliance improves with this treatment regime, thereby decreasing the treatment breaks and improving local tumor control. The primary site of tumor, AJCC stage of disease and number of cycles of concurrent chemotherapy are important prognostic factors for improved disease free survival in patients with LAHNC. Overall treatment time is an important prognostic factor for the local tumor control in LAHNC.

\section{References}

Adelstein DJ, Li Y, Adams GL, et al (2003). An Intergroup phase III comparison of standard radiation therapy and two schedules of concurrent chemo-radiotherapy in patients with unresectable squamous cell head and neck cancer. $J$ Clin Oncol, 21, 92-8.

Baykara M, Buyukberber S, Ozturk B, Coskun U, Unsal DK, Demirci U, et al (2013). Efficacy and safety of concomitant chemoradiotherapy with cisplatin and docetaxel in patients with locally advanced squamous cell head and neck cancers. Asian Pac J Cancer Prev, 14, 2557-61.

Browman GP, Hodson DI, Mackenzie RG, Bestic N, Zuraw L (2001). Cancer care ontario practice guideline initiative head and neck cancer disease site group: choosing a concomitant chemotherapy and radiotherapy regimen for squamous cell head and neck cancer: A systematic review of the published literature with subgroup analysis. Head Neck, 23, 579-89.

Brizel DM, Esclamado R (2006). Concurrent chemoradiotherapy for locally advanced, nonmetastatic, squamous carcinoma of the head and neck: consensus, controversy, and conundrum. J Clin Oncol, 24, 2612-17.

Chaturvedi P, (2009). Head and neck surgery. J Can Res Ther, 5,143 .

Cox JD, Stetz J, Pajak TF (1995). Toxicity criteria of the radiation therapy oncology group (RTOG) and the European organization for research and treatment of cancer (EORTC). Int J Radiat Oncol Biol Phys, 31, 1341-6.

Dimri K, Pandey AK, Trehan R, Rai B, Kumar A (2013). Conventional radiotherapy with concurrent weekly cisplatin in locally advanced head and neck cancers of squamous cell origin - a single institution experience. Asian Pac J Cancer Prev, 14, 6883-88.

Fan KY, Gogineni H, Zaboli D, et al (2012). Comparison of acute toxicities in two primary chemoradiation regimens in the treatment of advanced head and neck squamous cell carcinoma. Ann Surg Oncol, 19, 1980-7.

Forastiere AA, Goepfert H, Maor M, et al (2003). Concurrent chemotherapy and radiotherapy for organ preservation in advanced laryngeal cancer. $N$ Engl J Med, 349, 2091-98.

Geeta SN, Padmanabhan TK, Samuel J, et al (2006). Comparison of acute toxicities of two chemotherapy schedules for head and neck cancers. J Cancer Res Ther, 2, 100-4.

Gupta T, Agarwal JP, Ghosh-Laskar S, Parikh PM, D’Cruz AK, Dinshaw KA (2009). Radical radiotherapy with concurrent weekly cisplatin in loco-regionally advanced squamous cell carcinoma of the head and neck: a single-institution experience. Head Neck Oncol, $1,17$.

Lasrado S, Moras K, Pinto GJO, Bhat M, Hegde S, Sathian B, et al. (2014). Role of concomitant chemoradiation in locally advanced head and neck cancers. Asian Pac J Cancer Prev, 15, 4147-52.

Marcu L, van Doorn T, Olver I (2003). Cisplatin and radiotherapy in the treatment of locally advanced head and neck cancer. 